\title{
Elastic Deformation in Ceria Nanorods \\ via a Fluorite-to-Rutile Phase Transition
}

Thi X. T. Sayle and Dean C. Sayle

DASSR, Cranfield University, Defence Academy of the United Kingdom, Shrivenham, SN6 8LA, United Kingdom, d.c.sayle@cranfield.ac.uk

\begin{abstract}
Atomistic simulations reveal that ceria nanorods, under uniaxial tension, can accommodate over 9\% elastic deformation. Moreover, a fluorite-to-rutile phase change occurs above $6 \%$ strain, which is reversible upon release of the stress. The elastic energy stored within the nanorod may find application in future 'nanogenerators'.
\end{abstract}

Keywords: Atomistic Simulation, Microstructure, Molecular Dynamics, nanogenerator.

Much effort has been applied to the study of one-dimensional nanomaterials, such as nanorods and nanotubes, because of their unique electrical $\left[{ }^{1}\right]$, chemical $\left[{ }^{2},{ }^{3}\right]$ and mechanical $\left[{ }^{4}\right]$ properties, compared to the parent bulk material, with applications spanning: catalysis, to energy storage. However, central to their application in a diverse range of applications is their mechanical durability and 'perceived' fragility. Such vulnerability may reflect a structural response to, for example, friction and wear, localised compressive or tensile strain attributed to heating/cooling, operational vibration and for intercalation hosts, used to store charge carriers such as Li ions in rechargeable batteries, structural collapse under charge/discharge cycles [5]. High mechanical strength, toughness and fracture resistance are central to the exploitation of nanomaterials in a range of applications spanning biomedical and dental $\left[{ }^{6}\right]$ to MEMS $\left[{ }^{7}\right]$. Accordingly, to help meet such demanding requirements, a fundamental (atomistic) understanding of the mechanical properties and processes of nanomaterials, such as strength and fracture mechanics is needed. However, direct measurement of mechanical load is difficult experimentally - requiring in-situ mechanical deformation testing $\left[{ }^{8}\right]$. Notwithstanding such challenges, Shokuhfar and co-workers measured the mechanical compressive properties of individual thin-wall and thick-wall $\mathrm{TiO}_{2}$ nanotubes directly [ $\left.{ }^{9}\right]$ and found that that the Young's modulus of titanium dioxide nanotubes depended upon the diameter and wall 
thickness of the nanotube and is in the range of 23-44 GPa; the thin-wall nanotubes collapsed at 1.0 to $1.2 \mathrm{~N}$ during axial compression.

Atomistic computer simulation can be used to simulate mechanical deformation and is well-placed to complement experiment because the simulations are comparatively easier - molecular graphics can be used to explore the structural transformation during loading. For example, Xiong and co-workers explored uniaxial tension on $\mathrm{MgO}$ nanorods using molecular dynamics simulation, which revealed the atomic-scale mechanism of the deformation and failure process during tension $\left[{ }^{10}\right]$. In particular, they observed increased ductility at reduced strain rates. However, the difficulty associated with simulation is the generation of a structural model that is sufficiently realistic in that the results generated using the model are of value to experiment. Specifically, the nanorods are unlikely to be structurally perfect; rather they will contain microstructural features, such as morphology and surfaces exposed, grain-boundaries, dislocations and point defects. Indeed, Koh and Lee predicted that the tensile strength of platinum nanowires can change by up to $50 \%$ by altering the cross-sectional shape of the nanowire $\left[{ }^{11}\right]$. Moreover, is well known that the (measured) strength of a material is about two orders of magnitude less than its estimated ideal strength. This is attributed to the presence of microstructural features - specifically dislocations, which provide vehicles for plastic deformation. On the other hand nanomaterials can sustain stresses more that a tenth of their ideal strengths $\left[{ }^{12}\right]$ because they are less able to retain a dislocation population within their (size-constrained) structure compared to their bulk counterpart $\left[{ }^{13}\right]$.

To better understand the unique mechanical properties of nanomaterials, we simulated a nanorod of microstructural ceria under mechanical load. To generate such models we used simulated amorphisation and crystallisation $\left[{ }^{14}\right]$, fig. 1 , which is a simulation strategy capable of introducing variety of microstructural features into an atomistic model, including for example: surfaces and morphology, point defects and clusters, dislocations and grain-boundaries. Ceria is a prototypical, chemically reactive ceramic oxide, which has been exploited extensively in a wide range of applications including: fuel cells $\left[{ }^{15}\right]$, catalysts $\left[{ }^{16}\right]$, nanoabrasives $\left[{ }^{17}\right]$ and sensor $\left[{ }^{18}\right]$. 


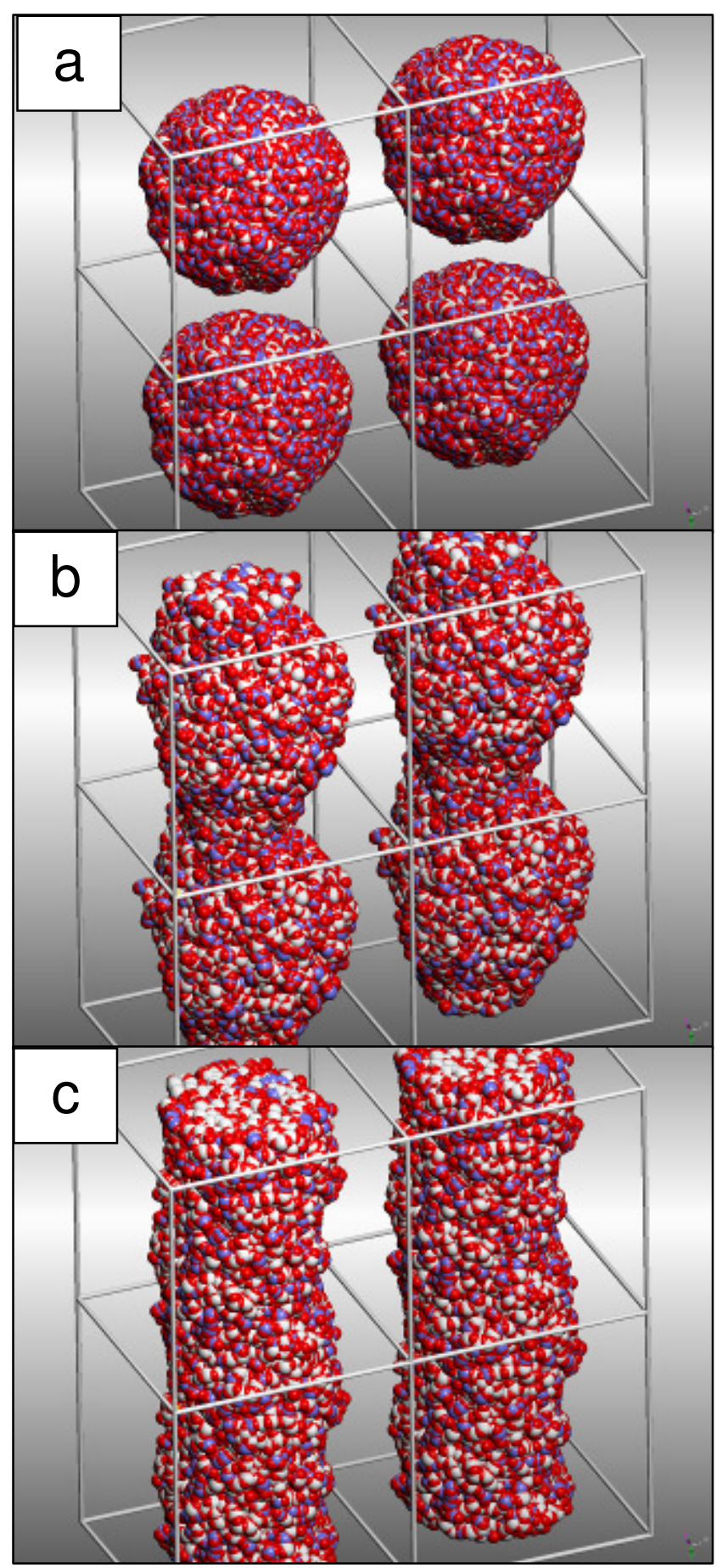

Figure 1 Strategy used to generate atomistic models for nanorods. (a) amorphous nanoparticles positioned into simulation cell; (b) agglomeration of neighbouring nanoparticles in one dimension; (c) evolution of nanorod topography; the nanorod is then crystallised. Atom positions are represented by white and blue spheres (cerium) and red spheres (oxygen). The blue spheres are used to show the high mobility of the ions within the molten phase. 


\section{Results and Discussion}

The structure of the crystalline $\mathrm{CeO}_{2}$ nanorod is shown in fig. 2. The rod is about $10 \mathrm{~nm}$ in diameter, exposes $\{111\}$ and $\{100\}$ surfaces facilitating a hexagonal cross-section and extends along the [110] direction in accord with experiment $\left[{ }^{19}\right]$, fig 2(b,c,d). Experimentally, nanorods with [210] as the principal axis have also been synthesised $\left[{ }^{20}\right]$. We found that by crystallising the nanorod at 3400 instead of $3750 \mathrm{~K}$ yielded a model of a nanorod that extended along [210].

Close inspection of the atomistic structure of the nanorod, fig 2(a) using molecular graphics, revealed a complex array of steps, edges and corners on the surface together with point defects that evolved both on the surface and in the bulk regions of the nanorod including cerium and oxygen vacancies and vacancy clusters. Such microstructural features are observed experimentally $\left[{ }^{16}\right]$. In particular, similar to real nanorods, our model is not simply a rod of ceria cleaved from the bulk material; rather it comprises a rich microstructure, which is an important feature of the model because this is likely to influence profoundly the mechanical strength and thus enable the model to display behaviour, which is more realistic compared to the real material.

The behaviour of the nanorod under mechanical load is shown in fig 3 and reveals that the yield strain is 0.066 with an associated tensile strength of about $18 \mathrm{GPa}$. The stress-strain trace is curved, which reflects a reduced Youngs modulus with increased strain; a straight line is shown as a visual aid. Inspection of the nanorod during tensile strain reveals that 'crack' formation emanates from the surface, fig. 4(a). However, surprisingly, upon increased strain, the $\mathrm{CeO}_{2}$ at the crack region undergoes a polymorphic transformation from fluorite to rutile, fig. 4(b). Moreover rather than suffering complete fracture, the nanorod maintains mechanical strength for a further 0.03 strain, at which point the simulation was stopped. Similar to a 'concertina', the nanorod accommodates the strain via this phase change; images showing more clearly the fluorite and rutile-structured $\mathrm{CeO}_{2}$ are shown in fig. 4 (c); a view looking perpendicular to the structure in $4(c)$ is shown in $4(d)$. 


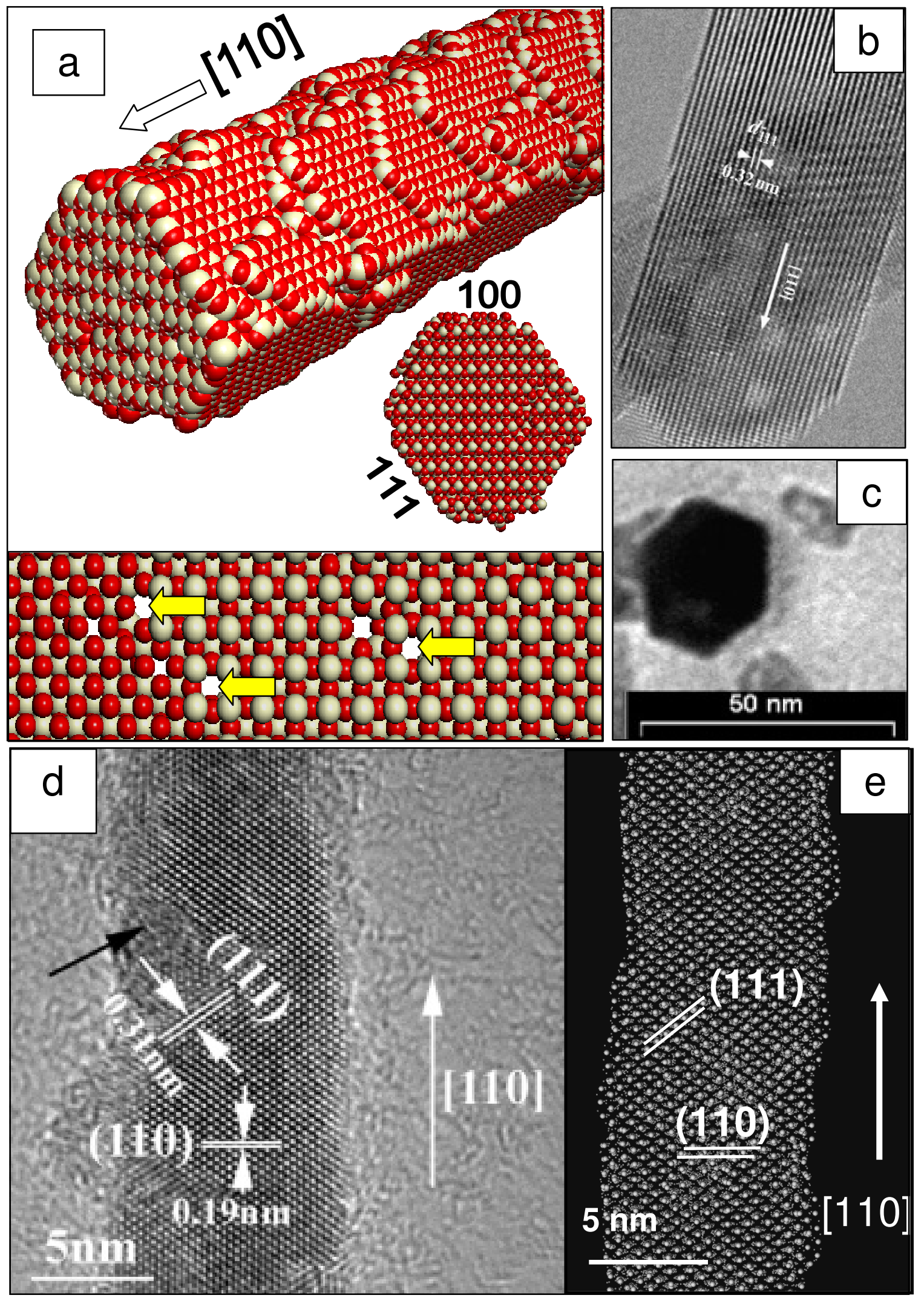

Figure 2 Models of the ceria nanorod compared with experiment: (a) atomistic model, top: perspective view; middle: view looking along [110] revealing the hexagonal profile of the nanorod; bottom: slice cut through the nanorod revealing the Ce and $\mathrm{O}$ vacancies that have evolved in the nanorod during (simulated) crystallization. (b, c) HRTEM images taken from ref. [19]. (d) HRTEM showing the [110] direction taken from ref. [20]. (e) atomistic model to compare with (d). In (a) the atom positions are represented by white spheres (cerium) and red spheres (oxygen). In (e) only the cerium atoms are shown. 

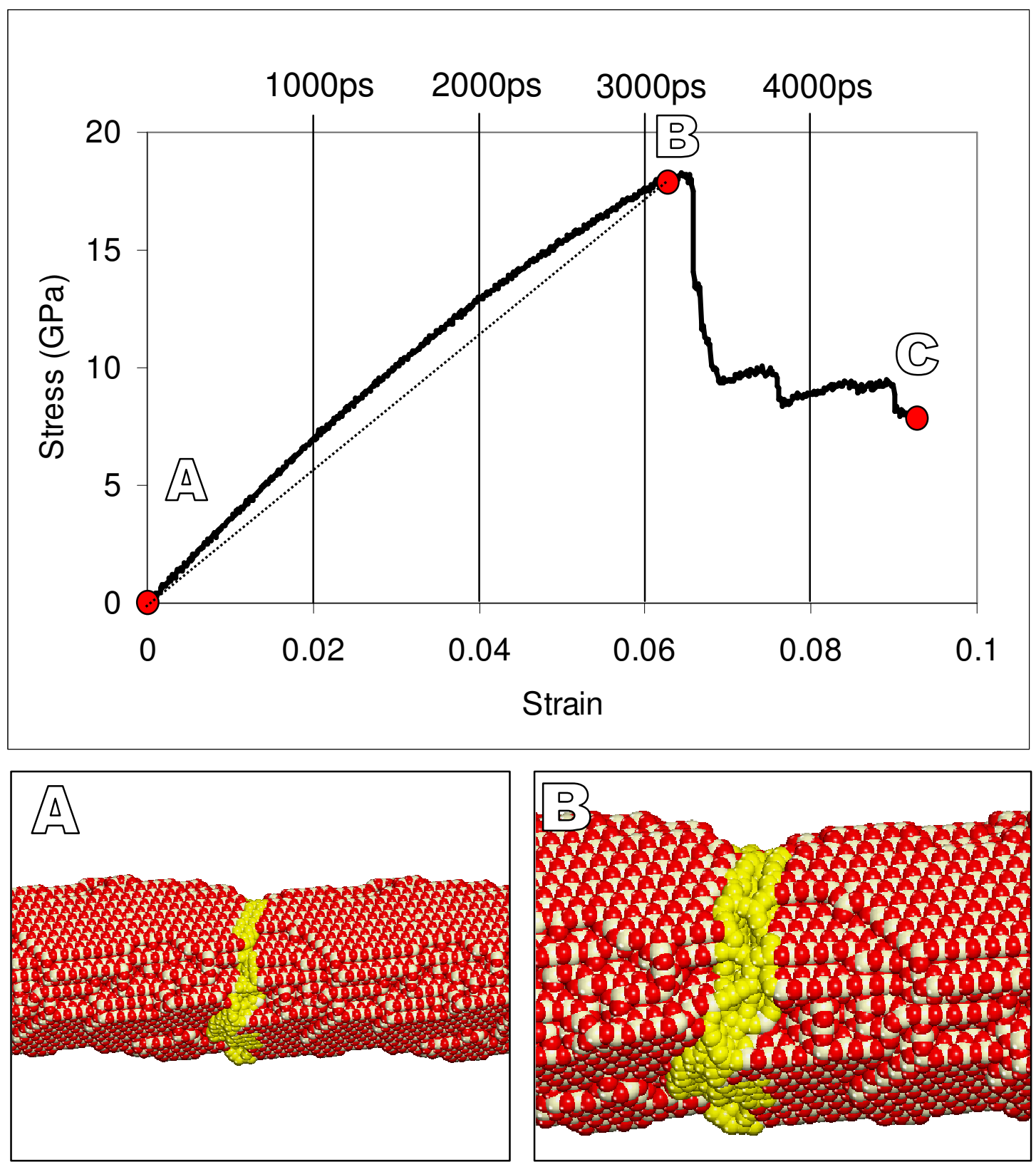

Figure 3 Top: Stress-strain curve, calculated for a ceria nanorod under tension; the secondary x-axis shows simulation time. Bottom: atomistic structures of the nanorod at the start of the simulation (A) and after 3212.5ps (B). Cerium is coloured white and oxygen is red; atoms coloured yellow indicate, more clearly, the approximate location of the 'crack'. 

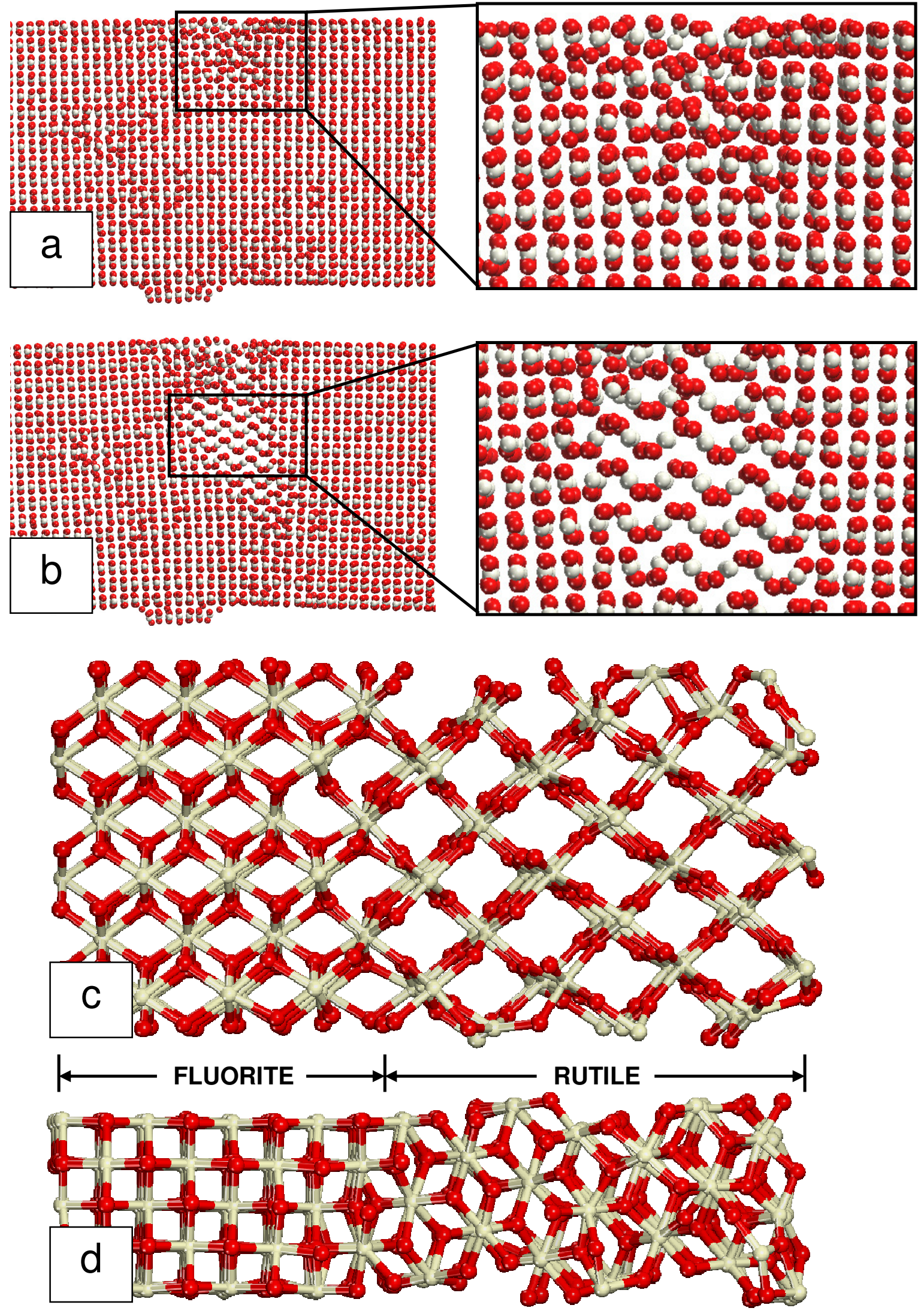

Figure 4 Atomistic structure of the ceria nanorod under tension. (a) after 3187 ps (strain 0.0637) (b, c, d) after 3212.5 ps (strain 0.0643); the structures can be usefully correlated with the stress-strain trace shown in fig. 3. Cerium is coloured white and oxygen is red. $(\mathrm{a}, \mathrm{b})$ sphere model representations of the atom positions, (c,d) ball and stick model representations. 
In fig 5, a region near the 'crack' is enlarged to reveal more clearly the mechanism underpinning the fluorite-rutile phase transformation. In particular, 5(a) shows the atomistic structure of a region of the $\mathrm{CeO}_{2}$ nanorod $(3200 \mathrm{ps}$, strain $=0.064)$ that conforms to the fluorite structure, which transforms to rutile after a further 12.5ps, fig. 5(b). Side views of 5(a) and (b) are shown in 5(c) and (d) respectively.

Inspection of the nanorod after the strain had been released at the end of the uniaxial tension simulation (0.093 strain, point $\mathrm{C}$ on fig. 3) revealed that it returns to its original configuration, figure 6; domains of $\mathrm{CeO}_{2}$ conforming to the rutile polymorph transform back to fluorite. In particular, the simulations suggest that the nanorod undergoes elastic deformation up to about 0.09 strain, which includes a fluorite-to-rutile phase change.

Further simulations, where the nanorod was tensioned uniaxially beyond a strain of 0.09 , revealed that the nanorod deforms plastically. In particular, regions in the vicinity of the crack that conform to the rutile polymorph, transform structurally back to the fluorite structure. The structure of the nanorod after 0.12 strain is shown in fig. 7. Upon release of the strain, the nanorod does not return to its original configuration.

Animations of the ceria nanorod under tensile strain, showing more clearly the mechanism underpinning the fluorite-rutile phase transformation, are available in supporting information. 

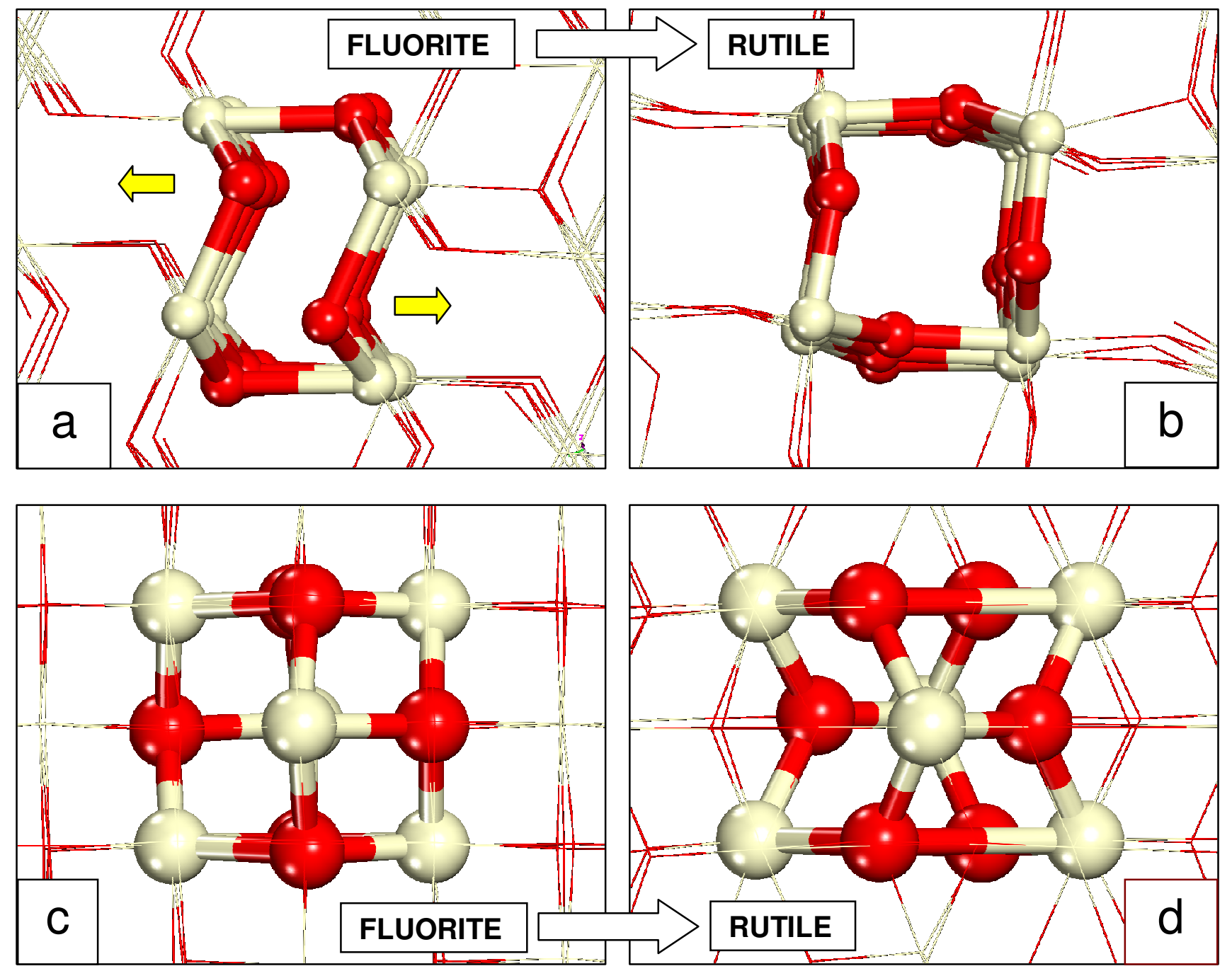

Figure 5 Atomistic structure of the ceria nanorod showing more clearly the fluorite-rutile phase transformation. (a) fluorite structure (3200ps, 0.064 strain), (b) rutile structure (3212.5ps, 0.0643 strain), (c) side view of (a), (d) side view of (b). 

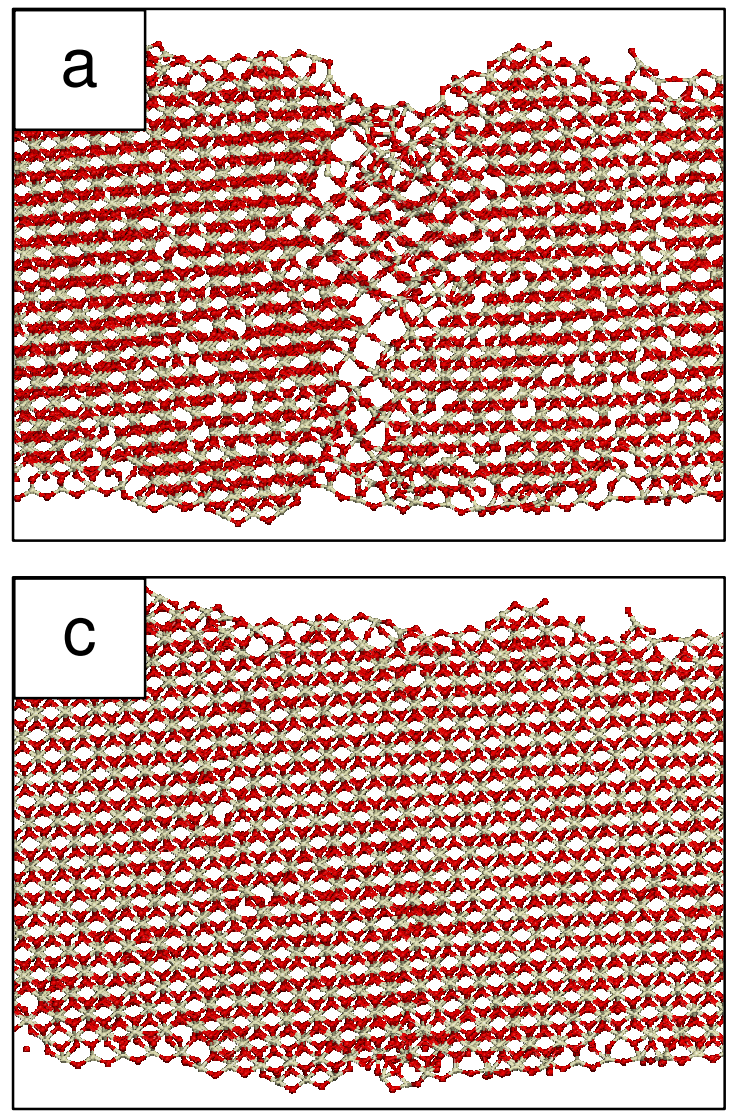
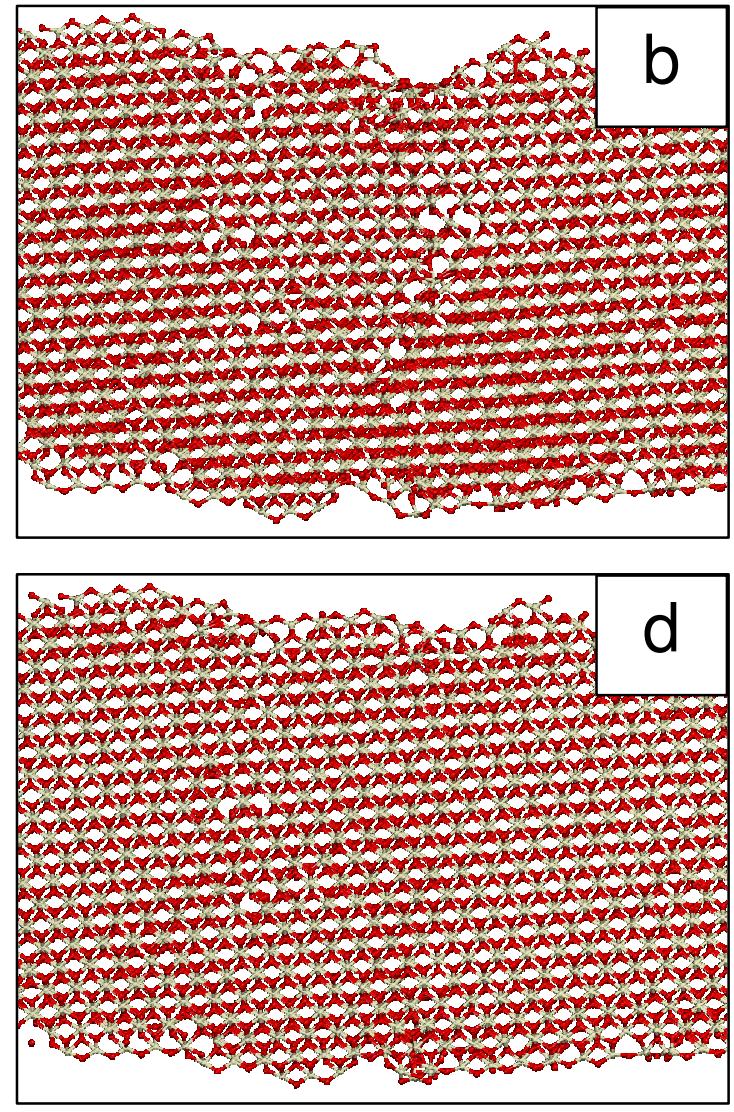

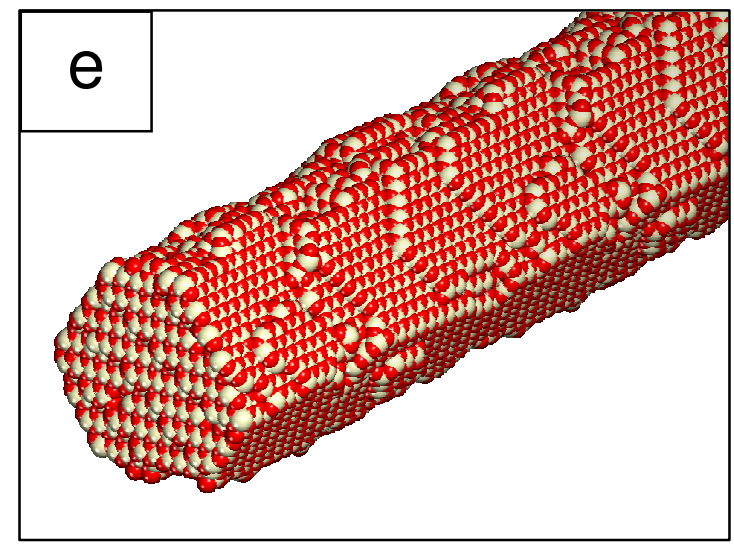

Figure 6. Dynamical structure of the $\mathrm{CeO}_{2}$ nanorod, at a (tensile) strain of 0.093 , after the stress has been relaxed. (a) starting structure, 0ps; (b) after 12.5ps; (c) after 25ps; (d) after 1200ps; (e) the same as (d) but with perspective view of the nanorod. Cerium is coloured white and oxygen is red. 

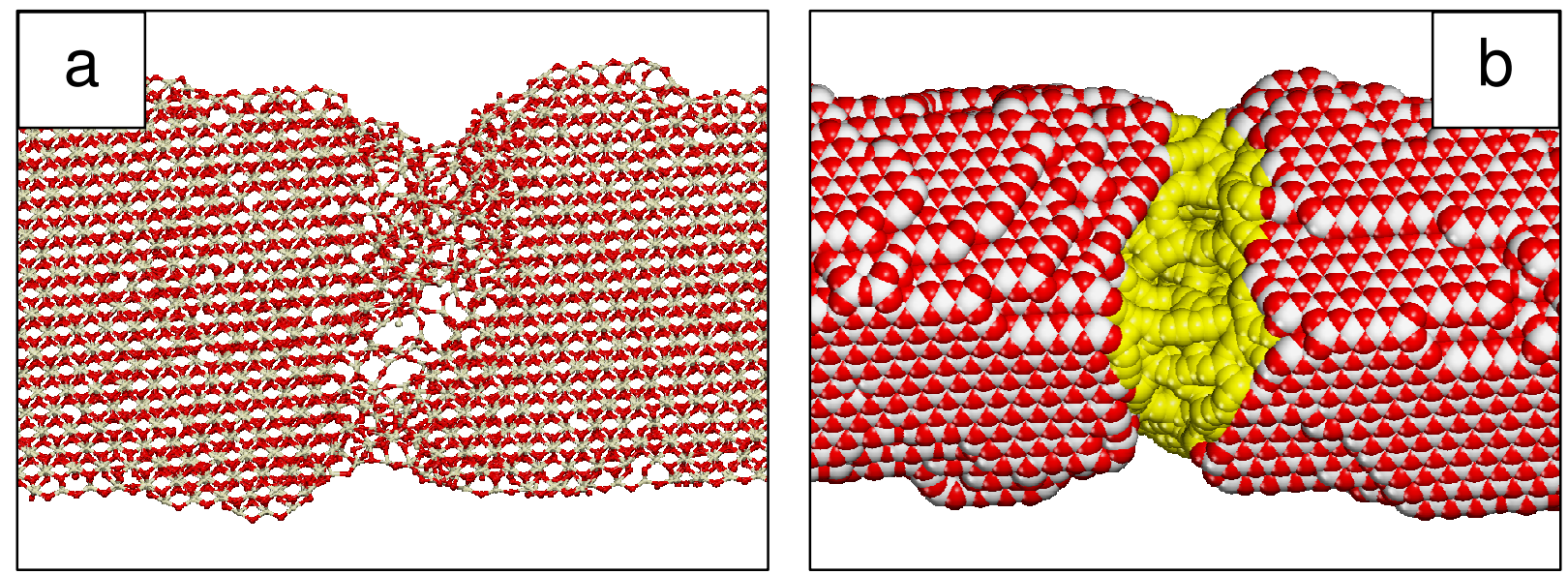

Figure 7 Structure of the nanorod after 0.12 strain. (a) slice cut through the nanorod revealing more clearly the crystal structure; (b) sphere model representation. Cerium is coloured white and oxygen is red. 
Changes in atomistic structure have been reported for nanorods under strain. In particular, in a gold nanowire, simulated under tension, a c.c.p. to b.c.t.1 packing of the metal atoms was predicted $\left[{ }^{21}\right]$; amorphisation of $\mathrm{SiC}$ nanowires was observed under deformation $\left[{ }^{22}\right]$; rutile-to-fluorite structural transformations in $\mathrm{SnO}_{2}$ nanorods and nanobelts under 35GPa compression have also been observed $\left[{ }^{23}\right]$. Crucially, the authors observed that the behaviour of the nanomaterial was (sometimes profoundly) different compared to the parent (bulk) material. Moreover, Agrawal and co-workers reported a wurtzite to bodycentred tetragonal phase change in $\mathrm{ZnO}$ nanowires $\left[{ }^{24}\right]$. We therefore propose that a fluorite-to-rutile phase transformation in $\mathrm{CeO}_{2}$ nanorods under tension as a precursor to ductile fracture is not unreasonable. In particular, rutile is less dense than fluorite and therefore this phase change offers a mechanism for relieving the strain on the nanorod; we await experimental confirmation. The elastic energy, stored in the ceria nanorod associated with the reversible fluorite-rutile phase change, may find possible application in the field of nanogenerators $\left[{ }^{25}\right]$ with reversible phase changes providing vehicles for nano-energy storage.

\section{Conclusion}

Most bulk ceramic materials, such as ceria $\left(\mathrm{CeO}_{2}\right)$ are brittle, which can impact severely upon their exploitation in a variety of applications. However, we predict that $\mathrm{CeO}_{2}$ nanorods deform elastically up to $9 \%$ tensile strain. Moreover, above $6 \%$ tensile strain, the $\mathrm{CeO}_{2}$ nanorod undergoes a fluorite-to-rutile phase transformation. Upon release of the stress, the nanorod returns to its original configuration; domains of rutile-structured $\mathrm{CeO}_{2}$ transform back to the fluorite polymorph.

\section{Acknowledgement}

EPSRC: EP/H001220, EP/H001298, EP/H005838.

Supporting Information Available: S1: animation showing a ceria nanorod undergoing a fluorite-rutile phase transformation; S2: animation showing the fracture of a ceria nanorod. This material is available free of charge via the Internet at http://pubs.acs.org.

\section{Methods}

All calculations, presented in this study, are based upon the Born model of the ionic solid, in which a charge is assigned to each atom and the long-range attractive interactions between these charged ions are balanced by short-range repulsive (electron-electron) interactions. Model parameters, used to describe $\mathrm{CeO}_{2}$, were taken from ref. $\left[{ }^{26}\right]$ and have been reported previously to model the structure of nanoparticles $\left[{ }^{17}\right]$, nanorods $\left[{ }^{27}\right]$ and nanotubes $\left[{ }^{28}\right]$ and for calculating important properties including oxygen mobility $\left[{ }^{28}\right]$, defect formation energy $\left[{ }^{26}\right]$. Accordingly, we suggest that such potential are well suited this present study. 
The DL_POLY code was used to perform all the molecular dynamics (MD) simulations[ $\left.{ }^{29}\right]$. All simulations were performed using three-dimensional periodic boundary conditions. Molecular graphics were performed using VMD $\left[{ }^{30}\right]$ and Materials Studio.

To generate a ceria nanorod, a 'cube' of $\mathrm{CeO}_{2}$, comprising 15972 atoms (5324 Ce, $10648 \mathrm{O}$ ), was cut from the parent material and the system melted by applying constant volume MD simulation at high temperature $(8000 \mathrm{~K})$; the simulation cell size was sufficiently large to ensure the nanoparticle does not feel any (repulsive or attractive) interaction from its periodic neighbours (fig 1a). The size of the simulation cell was then reduced in one dimension to enable the nanoparticle to interact and agglomerate with its periodic neighbours (fig. 1b) $\left.{ }^{27}\right]$. MD simulation, performed on the system for $1000 \mathrm{ps}$ at $8000 \mathrm{~K}$, facilitated the evolution of the nanorod (fig 1c). The nanorod was then crystallized by performing MD simulation at $3750 \mathrm{~K}$ for sufficient duration to converge the energy. Molecular graphics was used to examine the atomistic structure; nanorods that comprised dislocations or grain-boundaries were discarded because they are likely to weaken the nanorods because dislocations are vehicles for plastic deformation; rather nanorods comprising dislocations and/or grain-boundaries will be considered in a future study.

The mechanical properties of the nanorod were calculated by equilibrating the system to the target temperature by performing constant pressure MD simulation at $300 \mathrm{~K}$ for $100 \mathrm{ps}$ with $25 \mathrm{ps}$ equilibration, prior to simulating tensile and compressive strain, which was achieved by sequential scaling of the atom coordinates and performing constant volume MD simulation at $300 \mathrm{~K}$; a strain rate of about $10^{7} \mathrm{~s}^{-1}$ was attained.

\section{References}

\footnotetext{
${ }^{1}$ Xia, Y., Yang, P., Sun, Y., Wu, Y., Mayers, B., Gates, B., Yin, Y., Kim, F. \& Yan, H. Q Onedimensional nanostructures: synthesis, characterization, and applications. Adv. Mater., 15, 353 (2003).

${ }^{2}$ Zhou, K., Wang, X., Sun, X., Peng, Q. \& Li, Y. Enhanced catalytic activity of ceria nanorods from welldefined reactive crystal planes. J. Catal., 229, 206-212 (2005)
} 
${ }^{3}$ Liu, X., Zhou, K., Wang, L., Wang, B. \& Li. Y. Oxygen Vacancy Clusters Promoting Reducibility and Activity of Ceria Nanorods. J. of Am. Chem. Soc., 131, 3140- (2009).

${ }^{4}$ Riaz, M., Fulati, A., Amin, G., Alvi, N., Nur, O. \& Willander, M. Buckling and elastic stability of vertical $\mathrm{ZnO}$ nanotubes and nanorods. J. of Appl. Phys. 106, (2009).

${ }^{5}$ Debart, A., Paterson, A., Bao, J. \& Bruce, P. Alpha - MnO2 nanowires: A catalyst for the O-2 electrode in rechargeable lithium batteries. Angew. Chem. Int. Edit., 47, 4521-4524 (2008).

${ }^{6}$ Benzaid, R., Chevalier, J., Saddaoui, M., Fantozzi, G., Nawa, M., Diaz, L. \& Torrecillas, R. Fracture toughness, strength and slow crack growth in a ceria stabilized zirconia-alumina nanocomposite for medical applications. Biomaterials, 29, 3636-3641 (2008).

${ }^{7}$ Evans, A., Bieberle-Hutter, A., Rupp, J. \& Gauckler, L. Review on microfabricated micro-solid oxide fuel cell membranes. J. Power Sources 194, 119-129 (2009).

${ }^{8}$ Lockwood, A. \& Inkson, B. In situ TEM nanoindentation and deformation of Si-nanoparticle clusters. J. of phys. D Appl. Phys., 42 (2009).

${ }^{9}$ Shokuhfar, T. Arumugam, G.K., Heiden, P. A., Yassar, R. S. and Friedrich, C., Direct Compressive Measurements of Individual Titanium Dioxide Nanotubes, ACS Nano., 2009, In Press.

${ }^{10}$ Xiong, L., Chen, Y. \& Lee, J. Atomistic measure of the strength of $\mathrm{MgO}$ nanorods. Theor. Appl. Fract. Mec., 46, 202-208 (2006).

${ }^{11}$ Koh, S. \& Lee, H. Effects of Cross-Sectional Shape and Temperature on Mechanical Behavior of Platinum Nanowires. J. of Computational and Theoretical NanoScicence, 5, 1387-1397 (2008).

${ }^{12}$ Shan, Z., Adesso, G., Cabot, A., Sherburne, M., Asif, S., Warren, O., Chrzan, D. Minor, A. \& Alivisatos, A. Ultrahigh stress and strain in hierarchically structured hollow nanoparticles. Nature Materials 7, 947952 (2008).

${ }^{13}$ Suresh S. \& Li, J. Material science deformation of the ultra-strong. NATURE 456, 716-717 (2008).

${ }^{14}$ Sayle, T., Catlow, C., Maphanga, R., Ngoepe, P. \& Sayle, D. Generating MnO2 nanoparticles using simulated amorphization and recrystallization J. Am. Chem. Soc. 127, 12828-12837 (2005).

${ }^{15}$ Evans, A., Bieberle-Huetter, A., Rupp, J. L. M. and Gauckler, L. J. Review on microfabricated microsolid oxide fuel cell membranes. J. Power Sources, 194, 119-129, (2009)

${ }^{16}$ Esch, F., Fabris, S., Zhou, L., Montini, T., Africh, C., Fornasiero, P., Comelli, G. and Rosei, R.. Electron localization determines defect formation on ceria substrates, Science, 309, 752-755, (2005)

${ }^{17}$ Feng, X., Sayle D., Wang, Z., Paras, M., Santora, B., Sutorik, A., Sayle, T., Yang, Y., Ding, Y., Wang, X. \& Her, Y. Converting ceria polyhedral nanoparticles into single-crystal nanospheres, Science, 312, 15041508 (2006).

${ }^{18}$ Barreca, D., Gasparotto, A., Maccato, C., Maragno, C., Tondello, E., Comini, E. \& Sberveglieri, G. Columnar $\mathrm{CeO} 2$ nanostructures for sensor application. Nanotechnology, 18, (2007). 
${ }^{19}$ Vantomme, A., Yuan, Z. Y., Du, G. H. and Su, B. L., Surfactant-assisted large-scale preparation of crystalline $\mathrm{CeO} 2$ nanorods, Langmuir, 21, 1132-1135, (2005)

${ }^{20} \mathrm{Du}, \mathrm{N}$., Zhang, H., Chen, B., Ma, X. \& Yang, D. Ligand-free self-assembly of ceria nanocrystals into nanorods by oriented attachment at low temperature. J. Phys. Chem. C 111, 12677-12680 (2007).

${ }^{21}$ Diao, JK, Gall, K., \& Dunn, M. Surface-stress-induced phase transformation in metal nanowires. Nature Mat. 2, 656-660 (2003).

${ }^{22}$ Han, X., Zhang, Y., Zheng, K., Zhang, X., Zhang, Z., Hao, Y., Guo, X., Yuan, J. \& Wang, Z. Lowtemperature in situ large strain plasticity of ceramic SiC nanowires and its atomic-scale mechanism. Nano Letters, 7, 452-457 (2007).

${ }^{23}$ Dong, Z. H. and Song, Y., Chem. Phys. Lett., 480, 90-95 (2009).

${ }^{24}$ Agrawal, R., Peng, B. and Espinosa, H. D. Experimental-Computational Investigation of ZnO nanowires strength and fracture. Nano Letters, In press.

${ }^{25}$ Wang, ZL, Towards Self-Powered Nanosystems: From Nanogenerators to Nanopiezotronics. Adv. Funct. Mat., 18, 3555 (2008).

${ }^{26}$ Sayle, T., Parker, S. \& Catlow, C. The role of oxygen vacancies on ceria surfaces in the oxidation of carbon monoxide. Surf. Sci., 316, 329-336 (1994).

${ }^{27}$ Sayle, D., Feng, X., Ding, Y., Wang, Z., Sayle, T. Simulating synthesis": Ceria nanosphere self-assembly into nanorods and framework architectures. J. Am. Chem. Soc. 129, 7924-7935 (2007).

${ }^{28}$ Martin, P., Parker, S., Sayle, D. \& Watson, G. Atomistic modeling of multilayered ceria nanotubes. Nano Letters 7, 543-546 (2007).

${ }^{29}$ Smith, W.; Forester, T. R. DL_POLY, copyright by the council for the Central Laboratory of the Research Councils, Daresbury Laboratory, Daresbury, Warrington, UK www.cse.clrc.ac.uk/msi/software/DL_POLY/

${ }^{30}$ Humphrey, W., Dalke, A. \& Schulten, K. VMD - Visual Molecular Dynamics", J. Molec. Graphics, 14, 33-38 (1996). 